\title{
Сравнительное исследование хромогранина А и хромогранина В у больных с нейроэндокринными опухолями желудка и поджелудочной железы
}

\author{
Н.В. Любимова, Ю.С. Тимофеев, А.В. Лебедева, Н.Е. Кушлинский \\ ФГБУ «Национальный исследовательский медицинский центр онкологии им. Н.Н. Блохина» Минздрава России; Россия, \\ 115478 Москва, Каширское шоссе, 24
}

Конта кты : Нина Васильевна Любимова biochimia@yandex.ru

Введение. Иммуноферментный анализ биохимических маркеров является одним из важнейших методов диагностики нейроэндокринных опухолей (НЭ0). Общепризнанным маркером данной патологии служит хромогранин А (ХгА), а комплементарным - хромогранин В (ХгВ).

Цель исследования - изучить ХгВ в качестве дополнительного к ХгА биохимического маркера в сыворотке крови больных с НЭО желудка и поджелудочной железы.

Материалы и методы. Были обследованы 79 пациентов с НЭО желудка $(n=14)$ и поджелудочной железы $(n=65)$, а также 42 практически здоровых человека, которые вошли в контрольную группу. Хромогранин А и хромографин В определяли методом иммуноферментного анализа с помощью тест-систем Human Chromogranin B (USCN, Kитай) и Chromogranin A NEOLISA (Eurodiagnostica, Швеция).

Результаты. Уровни ХгВ в группе НЭО желудка и поджелудочной железы были значимо выше, чем в контрольной группе. Концентрация ХгВ не зависела от степени распространенности процесса и биологической активности новообразования. ROC-анализ показал, что в общей группе НЭO относительно группы контроля значение площади под кривой (AUC) для ХгB составило 0,869, а для ХгA - 0,82. По результатам статистического анализа в общей группе больных НЭО при изолированном использовании ХгА и ХгВ имели сопоставимую диагностическую чувствительность, которая повышалась при их совместном применении до 82,5 \%. В группе больных НЭО с низким уровнем ХгА (<100 нг/мл) в 53,6 \% случаев наблюдалась концентрация ХгВ выше порогового уровня (>15,7 нг/мл).

Заключение. Комплексное применение ХгВ и ХгА при НЭО поджелудочной железы и желудка способно повысить диагностическую эффективность биохимической диагностики. Полученные данные подтверждают значение ХгВ в качестве комплементарного биомаркера НЭО.

Ключевые слова: хромогранин A, хромогранин В, биохимический маркер, нейроэндокринная опухоль, диагностика

Для цитирования: Любимова Н.В., Тимофеев Ю.С., Лебедева А.В., Кушлинский Н.Е. Сравнительное исследование хромогранина А и хромогранина В у больных с нейроэндокринными опухолями желудка и поджелудочной железы. Успехи молекулярной онкологии 2021;8(3):8-13. DOI: 10.17650/2313-805X-2021-8-3-8-13.

\section{Comparative assay of chromogranin A and chromogranin B in patients with pancreatic and gastric neuroendocrine tumors}

\author{
N.V. Lyubimova, Yu.S. Timofeev, A. V. Lebedeva., N.E. Kushlinskii \\ N.N. Blokhin National Cancer Research Center, Ministry of Health of Russia; 24 Kashirskoe Shosse, Moscow 115478, Russia
}

Cont a ct s: $\quad$ Nina Vasilyevna Lyubimova biochimia@yandex.ru

Introduction. Immunoenzyme assay of biochemical markers is one of the most important methods for examination of patients with neuroendocrine tumors (NETs). Along with the generally accepted NET marker chromogranin A (CgA), another member of the granin family, chromogranin $B(\mathrm{CgB})$, can serve as a complementary marker.

Objectives. Analysis of $\mathrm{CgB}$ as an additional to $\mathrm{CgA}$ biochemical marker in the blood serum of patients with gastric and pancreatic neuroendocrine tumors. 


\begin{abstract}
Materials and methods. We examined 79 patients with gastic $(n=14)$ and pancretic $(n=65)$ NETS, and 42 particularly healthy people, who were included in the control group. $\mathrm{CgB}$ and $\mathrm{CgA}$ were determined with ELISA method using the Human Chromogranin B (USCN, China) and Chromogranin A NEOLISA (Eurodiagnostica, Sweden) test systems.

Results. CgB levels in gastric and pancreatic NETs were significantly higher than in control group. $\mathrm{CgB}$ concentrations were independent of tumor spread and its biological activity. ROC analysis in common group of NETs relative to control group showed $A U C$ for $\mathrm{CgB}=0.869$ and for $\mathrm{CgA} \mathrm{AUC}=0.82$. According to results in common group of NET patients when used isolated, $\mathrm{CgA}$ and $\mathrm{CgB}$ have comparable diagnostic sensitivity, which increases in complex use to $82.5 \%$. In the group of NET patients with low levels of $\mathrm{CgA}(<100 \mathrm{ng} / \mathrm{ml})$, an increase in $\mathrm{CgB}$ concentration above the cut-off level $(>15.7 \mathrm{ng} / \mathrm{ml}$ ) was observed in $53.6 \%$ of cases.

Conclusion. The combination of $\mathrm{CgB}$ and $\mathrm{CgA}$ in gastric and pancreatic NETs could increase the diagnostic efficacy of biochemical diagnostics. The received data confirms the significance of $\mathrm{CgB}$ as a complementary biomarker of NETs.
\end{abstract}

Key words: chromogranin A, chromogranin B, biochemical marker, neuroendocrine tumors, diagnosis

For citation: Lyubimova N.V., Timofeev Yu.S., Lebedeva A.V., Kushlinskii N.E. Comparative assay of chromogranin A and chromogranin B in patients with pancreatic and gastric neuroendocrine tumors. Uspekhi molekulyarnoy onkologii = Advances in Molecular Oncology 2021;8(3):8-13. (In Russ.). DOI: 10.17650/2313-805X-2021-8-3-8-13.

\section{ВВЕДЕНИЕ}

Исследование уровней циркулирующих маркеров при нейроэндокринных опухолях (НЭО) является одним из важных методов неинвазивной диагностики. Это обусловлено тем, что для данных новообразований характерна специфическая секреторная активность, ассоциированная с гиперпродукцией целого ряда пептидов, гормонов и биогенных аминов, многие из которых формируют определенную клиническую симптоматику [1-4]. Однако в большинстве случаев специфические симптомы при НЭО появляются не сразу, а спустя несколько лет после возникновения первичной опухоли. При этом на момент верификации диагноза пациент зачастую имеет распространенную форму заболевания с метастатическим поражением печени [4].

В настоящее время выделяют 2 основные группы биохимических маркеров НЭО: универсальные, не связанные с определенной симптоматикой, и специфические, ассоциированные с тем или иным гиперфункциональным синдромом [1-6]. Среди универсальных биомаркеров наибольшее значение имеет хромогранин А (ХгА) - белок семейства гранинов, который в настоящее время считается основным маркером НЭО [7-11]. Несмотря на то, что ХгА играет большую роль в биохимической диагностике НЭО различных локализаций, он имеет такие ограничения, как недостаточная чувствительность при локализованных формах заболевания, включая НЭО поджелудочной железы, а также зависимость концентрации в сыворотке крови от наличия сопутствующих заболеваний и получаемых препаратов [2, 4, 5, 12]. Таким образом, необходим поиск дополнительных биохимических маркеров, которые смогут выявить нейроэндокринную природу опухолевого процесса у больных с нормальной секрецией ХгА. Кандидатом на роль такого комплементарного маркера может стать другой член семейства гранинов - хромогранин В (ХгВ), который в отличие от ХгА недостаточно изучен [13-16]. Данная работа является продолжением нашего предыдущего исследования [17], в котором мы впервые начали изучать секрецию хромогранина В при НЭО поджелудочной железы. Исследование сывороточных концентраций белков семейства гранинов ХгА и ХгВ при нейроэндокринных неоплазиях различного типа позволит проанализировать потенциальную возможность их комплексного применения.

Цель исследования - изучить ХгВ в качестве дополнительного к ХгА биохимического маркера в сыворотке крови больных НЭО желудка и поджелудочной железы.

\section{МАТЕРИАЛЫ И МЕТОДЫ}

Мы обследовали 79 пациентов с НЭО желудка $(n=14)$ и поджелудочной железы $(n=65)$ в возрасте от 18 до 80 лет (медиана - 60,5 года), среди которых было больше женщин $(n=60)$. По результатам клинического и инструментального обследований у 46 (58,2 \%) больных выявлены метастазы в печени и у $24(30,4 \%)$ подтвержден карциноидный синдром. Верификация диагноза проводилась по данным морфологического исследования материала, полученного в результате биопсии или последующего оперативного вмешательства. Все пациенты на момент обследования не получали специфического лечения.

В группу контроля вошли 42 практически здоровых человека (без онкологических заболеваний) в возрасте от 18 до 85 лет (медиана - 55,0 лет), из них - 28 женщин и 14 мужчин.

Анализ биохимических маркеров проводился в сыворотке крови пациентов с НЭО и практически здоровых людей, взятой натощак из кубитальной вены. Для определения ХгВ применялась стандартизованная иммуноферментная тест-система Human Chromogranin B (USCN, Китай). Анализ ХгА проводился с помощью набора Chromogranin A NEOLISA (Eurodiagnostica, Швеция). Иммуноферментный анализ сыворотки крови выполнялся в полуавтоматическом формате на анализаторе BEP 2000 (Siemens, Германия). Анализ данных проведен с помощью пакетов статистических 
программ Statistica 10 (расчет достоверности различий по критерию Манна-Уитни, анализ корреляционных взаимосвязей по Спирмену) и SPSS (построение и анализ ROC-кривых).

\section{РЕЗУЛЬТАТЫ}

На 1-м этапе мы проанализировали результаты определения ХгВ и ХгА в сыворотке крови больных НЭО и практически здоровых людей, вошедших в контрольную группу (табл. 1).

Медиана концентрации ХгВ в сыворотке крови в общей группе НЭО была статистически значимо выше, чем в контрольной группе, и превышала ее в 2 раза. Анализ показал, что у больных НЭО желудка и поджелудочной железы уровни ХгВ были значимо выше, чем у практически здоровых людей ( $p=0,0001$ и $p=0,0000001)$. При этом медианы в группах НЭО желудка и поджелудочной железы не различались $(p=0,4)$.

Сравнительный анализ уровней ХгВ при различных клинических вариантах НЭО показал отсутствие зависимости этого маркера от степени распространенности процесса и биологической активности опухоли. Так, медианы уровней ХгВ как в группе НЭО с карциноидным синдромом, так и без него, были статистически значимо выше, чем в контрольной группе $(p=0,000002$ и $p=0,0000001)$, в то же время уровни ХгВ в различных группах больных НЭО не различались $(p=0,1)$. Важным моментом, подтверждающим наше предыдущее исследование [17], является относительная независимость значений ХгВ от степени распространенности опухолевого процесса. Медианы концентраций ХгВ достоверно превышали данный показатель в контрольной группе как при НЭО с метастатическим поражением печени ( $p=0,0000001)$, так и при локализованных формах заболевания $(p=0,000011)$, однако статистически значимых различий между уровнями этого маркера у пациентов с разной распространенностью процесса выявлено не было $(p=0,17)$.

Также мы проанализировали результаты определения уровня ХгА в исследуемых группах (см. табл. 1).

Таблица 1. Уровни хромогранина А и В в сыворотке крови пациентов с нейроэндокринными опухолями и в контрольной группе Table 1. Chromogranin A u B levels in the blood serum of patients with neuroendocrine tumors and in the control group

\begin{tabular}{|c|c|c|c|c|c|c|}
\hline \multirow[b]{2}{*}{$\begin{array}{l}\text { Групाпа } \\
\text { Group }\end{array}$} & \multicolumn{3}{|c|}{$\begin{array}{c}\text { Уровень хромогранина А (нг/мл) } \\
\text { Chromogranin A level (ng/ml) }\end{array}$} & \multicolumn{3}{|c|}{$\begin{array}{c}\text { Уровень хромогранина В (нг/мл) } \\
\text { Chromogranin B level (ng/ml) }\end{array}$} \\
\hline & $\begin{array}{c}\text { Медиана } \\
\text { (квартили) } \\
\text { Median } \\
\text { (quartiles) }\end{array}$ & $\begin{array}{l}\text { Минимум - } \\
\text { максимум } \\
\text { Minimum - } \\
\text { maximum }\end{array}$ & $\begin{array}{l}\text { Отличие } \\
\text { от групाы } \\
\text { контроля, } \boldsymbol{p} \\
\text { Difference from } \\
\text { control group, p }\end{array}$ & $\begin{array}{c}\text { Медиана } \\
\text { (квартили) } \\
\text { Median } \\
\text { (quartiles) }\end{array}$ & $\begin{array}{l}\text { Минимум - } \\
\text { максимум } \\
\text { Minimum - } \\
\text { maximum }\end{array}$ & $\begin{array}{l}\text { Отличие } \\
\text { от групाшы } \\
\text { контроля, } \boldsymbol{p} \\
\text { Difference } \\
\text { from control } \\
\text { group, p }\end{array}$ \\
\hline $\begin{array}{l}\text { Общая группа } \\
\text { нейроэндокринных опухолей } \\
\text { Common group of neuroendocrine } \\
\text { tumors }\end{array}$ & $\begin{array}{c}193 \\
(68,6-558)\end{array}$ & $16,5-39226$ & 0,0000001 & $\begin{array}{c}18,6 \\
(14,3-26,3)\end{array}$ & $5,9-47,9$ & 0,0000001 \\
\hline $\begin{array}{l}\text { Нейроэндокринные опухоли } \\
\text { желудка } \\
\text { Gastric neuroendocrine tumors }\end{array}$ & $\begin{array}{c}237 \\
(154-368)\end{array}$ & $89,7-1319$ & 0,000001 & $\begin{array}{c}17,5 \\
(12,7-22,9)\end{array}$ & $7,8-36,0$ & 0,0001 \\
\hline $\begin{array}{l}\text { Нейроэндокринные опухоли } \\
\text { поджелудочной железы } \\
\text { Pancreatic neuroendocrine tumors }\end{array}$ & $\begin{array}{c}154 \\
(59,5-559)\end{array}$ & $16,5-39226$ & 0,0000001 & $\begin{array}{c}19,4 \\
(14,7-26,7)\end{array}$ & $5,9-47,9$ & 0,0000001 \\
\hline $\begin{array}{l}\text { Нейроэндокринные опухоли } \\
\text { с карциноидным синдромом } \\
\text { Neuroendocrine tumors with carcinoid } \\
\text { syndrome }\end{array}$ & $\begin{array}{c}278 \\
(125-1367)\end{array}$ & $29,7-36217$ & 0,0000001 & $\begin{array}{c}16,8 \\
(12,6-23,1)\end{array}$ & $8,2-28,5$ & 0,000002 \\
\hline $\begin{array}{l}\text { Нейроэндокринные опухоли } \\
\text { без признаков карциноидного } \\
\text { синдрома } \\
\text { Neuroendocrine tumors without } \\
\text { carcinoid syndrome }\end{array}$ & $\begin{array}{c}177 \\
(62,2-368)\end{array}$ & $16,5-39229$ & 0,0000001 & $\begin{array}{c}19,6 \\
(15,6-27,6)\end{array}$ & $5,9-47,9$ & 0,0000001 \\
\hline $\begin{array}{l}\text { Нейроэндокринные опухоли } \\
\text { с метастазами в печени } \\
\text { neuroendocrine tumors with hepatic } \\
\text { metastasis }\end{array}$ & $\begin{array}{c}259 \\
(125-1107)\end{array}$ & $31,4-39226$ & 0,0000001 & $\begin{array}{c}20,8 \\
(14,8-27,5)\end{array}$ & $6,2-47,9$ & 0,0000001 \\
\hline $\begin{array}{l}\text { Нейроэндокринные опухоли } \\
\text { без метастазов в печени } \\
\text { Neuroendocrine tumors without } \\
\text { hepatic metastasis }\end{array}$ & $\begin{array}{c}100,8 \\
(62,1-284)\end{array}$ & $16,5-930,4$ & 0,000048 & $\begin{array}{c}17,6 \\
(12,8-21,5)\end{array}$ & $10,0-36,5$ & 0,000011 \\
\hline $\begin{array}{l}\text { Контрольная группа } \\
\text { Control group }\end{array}$ & $\begin{array}{c}54,1 \\
(45,5-68,4)\end{array}$ & $21,1-147$ & - & $\begin{array}{c}9,6 \\
(7,4-13,7)\end{array}$ & $3,2-20,2$ & - \\
\hline
\end{tabular}


Медианы ХгА как в общей группе НЭО (193 нг/мл), так и в группе НЭО желудка (237 нг/мл) и поджелудочной железы (154 нг/мл) статистически значимо $(p<0,0001)$ превышали данный показатель в контрольной группе (54,1 нг/мл). Дополнительный анализ выявил зависимость уровня ХгА от степени распространенности процесса: медиана концентраций этого хромогранина в группе НЭО с метастазами в печени (259 нг/мл) была выше, чем в группе с локализованными формами данного заболевания (100,8 нг/мл), при этом различия носили статистически значимый характер $(p=0,0054)$.

Для оценки диагностической эффективности ХгВ мы сравнили его с ХгА в общей группе. В нашей выборке положительная корреляционная зависимость уровней ХгА и ХгВ была статистически значимой $(p=0,0025)$, но имела слабо выраженную силу (коэффициент Спирмена = 0,33), что свидетельствует об относительной независимости данных маркеров и возможности их комплементарного использования. По результатам ROC-анализа в общей группе НЭО относительно группы контроля значение площади под кривой (AUC) для ХгВ составило 0,869 , а для ХгА 0,82 (см. рисунок).

При проведении ROC-анализа были получены пороговые значения сывороточных концентраций ХгВ и ХгА с использованием индекса Юдена $\left(\mathrm{J}_{\max }\right)$, которые применялись для последующей оценки диагностической эффективности этих маркеров по всем анализируемым в нашей работе группам. При пороговом уровне ХгВ 15,7 нг/мл $(\mathrm{J}=0,575)$ и диагностической специфичности, полученной в группе контроля, 89,5\% диагностическая чувствительность этого маркера в общей группе НЭО составила 68,8 \%. При пороговом уровне ХгА 100 нг/мл $(\mathrm{J}=0,616)$ диагностическая специфичность данного маркера в группе контроля со-

$\boldsymbol{a}$

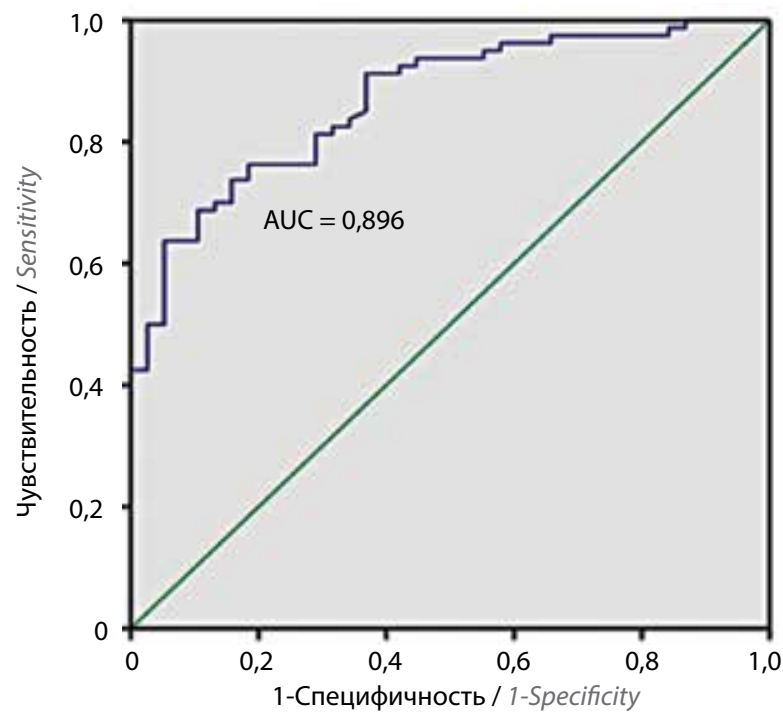

ставила 94,9 \%, а диагностическая чувствительность в группе больных НЭО - 66,7%. Результаты анализа диагностической чувствительности ХгВ и ХгА представлены в табл. 2.

Согласно данным таблицы, в общей группе НЭО при самостоятельном применении ХгА и ХгВ имеют сопоставимую диагностическую чувствительность. При совместном исследовании маркеров с учетом

Таблица 2. Диагностическая чувствительность хромогранина В и А при их изолированном и комплексном исследовании

Table 1. Diagnostic sensitivity of chromogranin B u A with isolated and a complex analysis

\begin{tabular}{|c|c|c|c|}
\hline \multirow[b]{2}{*}{$\begin{array}{l}\text { Группа } \\
\text { Group }\end{array}$} & \multicolumn{3}{|c|}{$\begin{array}{c}\text { Диагностическая } \\
\text { чувствительность, \% } \\
\text { Diagnostic sensitivity, \% }\end{array}$} \\
\hline & $\begin{array}{l}\text { Хромо- } \\
\text { гранин А } \\
\text { Chromo- } \\
\text { granin A }\end{array}$ & $\begin{array}{l}\text { Хромо- } \\
\text { гранин B } \\
\text { Chromo- } \\
\text { granin B }\end{array}$ & $\begin{array}{c}\text { Хромогра- } \\
\text { нин A + } \\
\text { хромогра- } \\
\text { нин B } \\
\text { Chromo- } \\
\text { granin A + } \\
\text { chromo- } \\
\text { granin B }\end{array}$ \\
\hline $\begin{array}{l}\text { Общая группа нейро- } \\
\text { эндокринных опухолей } \\
\text { Common group of } \\
\text { neuroendocrine tumors }\end{array}$ & 66,7 & 68,8 & 82,5 \\
\hline $\begin{array}{l}\text { Нейроэндокринные } \\
\text { опухоли желудка } \\
\text { Gastric neuroendocrine } \\
\text { tumors }\end{array}$ & 92,9 & 64,3 & 100,0 \\
\hline $\begin{array}{l}\text { Нейроэндокринные } \\
\text { опухоли поджелудочной } \\
\text { железы } \\
\text { Pancreatic neuroendocrine } \\
\text { tumors }\end{array}$ & 60,3 & 70,8 & 78,4 \\
\hline
\end{tabular}

б

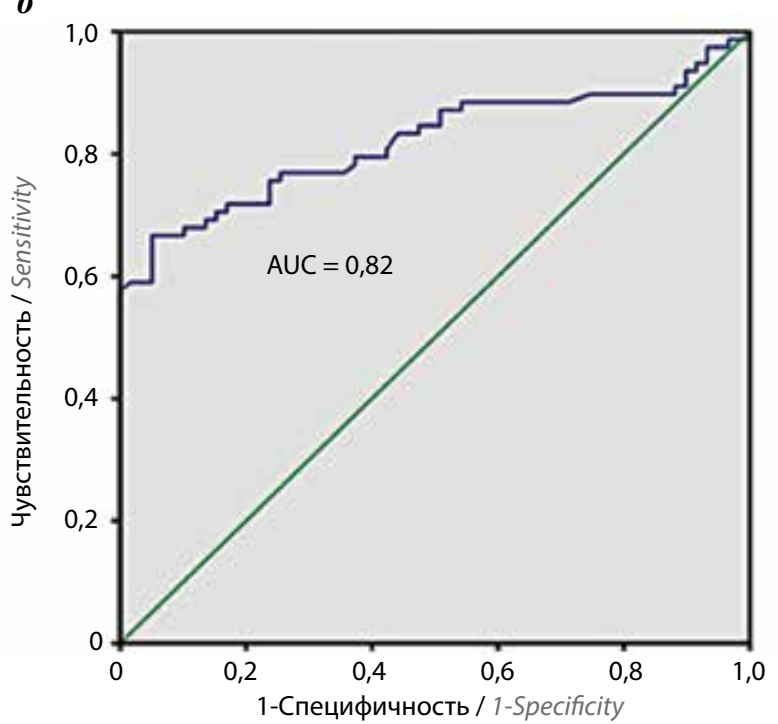

Результаты ROC-анализа диагностической эффективности ХгВ (а) и ХгА (б). ХгВ - хромогранин В; ХгA-хромогранин $A$

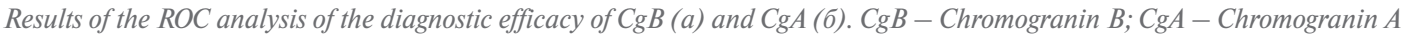


полученных нами пороговых уровней частота превышения порога хотя бы одного из данных маркеров достигала 82,5 \% в общей группе НЭО при диагностической специфичности, полученной в группе контроля, 80,9 \%. Для группы НЭО желудка чувствительность ХгА была выше, чем чувствительность ХгВ. При этом у всех пациентов наблюдалось повышение уровней одного или обоих маркеров, что позволяло достигнуть практически 100-процентной чувствительности с использованием полученных ранее пороговых уровней. В то же время при НЭО поджелудочной железы диагностическая чувствительность ХгВ была выше, чем ХгА. При этом повышение концентрации хотя бы одного маркера было выявлено у 78,4 \% больных НЭО поджелудочной железы. Дальнейшая оценка комплексного использования ХгА и ХгВ предполагает обследование большего количества пациентов с НЭО, что позволит провести детальный анализ данных.

Для дополнительной оценки комплементарности ХгВ к ХгА мы провели анализ частоты повышения сывороточной концентрации ХгВ в группе больных НЭО, имеющих низкие уровни ХгА (до 100 нг/мл), с помощью стандартного метода построения частотных таблиц (Frequency tables, Statistica 10). При этом в 53,6 \% случаев наблюдалось повышение концентрации ХгВ относительно порогового уровня (15,7 нг/мл), что позволяет использовать его в качестве биомаркера НЭО в тех случаях, когда уровень ХгА находится в пределах референсного интервала.

\section{ОБСУЖДЕНИЕ}

Согласно результатам исследования, комплексное исследование ХгА и ХгВ является эффективным способом повышения точности диагностики НЭО. Хромогранин А имеет наибольшую диагностическую чувствительность при распространенных формах заболевания, сопровождающихся метастатическим поражением печени и других органов [2, 4, 6, 11]. В то же время чувствительность данного маркера значительно ниже при биологически неактивных панкреатических НЭО [2, 8, 9], что обусловливает необходимость поиска более эффективного комплекса универсальных маркеров для повышения точности биохимической диагностики этого типа неоплазий.

В мировой литературе приводятся данные о возможности комплексного применения ХгА и ХгВ при гастроэнтеропанкреатических НЭО [13-16]. Первые сравнительные исследования различных белков семейства гранинов в качестве биохимических маркеров были проведены М. Stritsberg и соавт. [14]. На основе радиоиммунного анализа авторы выявили повышение циркулирующих уровней не только ХгА, но в большинстве случаев и ХгВ. Позже исследователи выявили, что диагностическая чувствительность ХгВ в общей группе НЭО не уступала ХгА, при этом уровень ХгВ не зависел от сопутствующей почечной патологии и приема ингибиторов протонной помпы [13].

Исследования в этом направлении были продолжены в 2017 г. японскими учеными М. Miki и соавт., которые для определения ХгВ больных НЭО поджелудочной железы использовали радиоиммунный метод [15]. Диагностическая чувствительность этого маркера при НЭО поджелудочной железы составила $73 \%$ (при специфичности 77 \%) и была сопоставима с полученными нами данными (70,8 \%), однако в нашей работе специфичность ХгВ была выше $(89,5 \%)$. Авторы пришли к выводу, что ХгВ имеет большой потенциал как маркер панкреатических НЭО ранних стадий без метастазов в печени. Это согласуется с нашими данными о независимости уровня маркера от распространенности процесса. Данное исследование продолжает начатую нами в нашей стране работу по изучению перспектив использования ХгВ в диагностике НЭО [17]. Полученные нами результаты на большей выборке больных НЭО поджелудочной железы и дополнительно пациентов с НЭО желудка согласуются с полученными ранее данными о потенциальной эффективности ХгВ как биологического маркера, комплементарного к ХгА.

\section{ЗАКЛЮЧЕНИЕ}

Комплексное применение ХгВ и ХгА при НЭО поджелудочной железы и желудка способно повысить диагностическую эффективность исследования этих маркеров. В соответствии с полученными нами данными уровень ХгВ не зависит от функциональной активности и распространенности новообразований, что позволяет использовать его для выявления нефункционирующих опухолей поджелудочной железы, при которых в 50 \% случаев наблюдаются низкий уровень ХгА и отсутствие секреции специфических маркеров, таких как вазоактивный интестинальный пептид, соматостатин, глюкагон. Дополнительный анализ группы больных с низкими уровнями ХгА показал, что более чем у 50 \% пациентов выявляется гиперсекреция ХгВ. Это расширяет возможности выявления нейроэндокринной природы процесса у значимой доли больных с нормальными уровнями ХгА.

Результаты нашего исследования подтверждают значимость использования ХгВ и ХгА в качестве биомаркеров в целях оптимизации точности биохимической диагностики НЭО. В то же время остаются неизученными значения ХгВ при других локализациях (легкие, толстая и тонкая кишка) и его перспективы как универсального маркера опухолей нейроэндокринной природы. 


\section{$\begin{array}{lllllllllllllllllllll}\text { Л } & \text { И } & \text { T } & \text { E } & \text { P } & \text { A } & \text { T } & \text { Y } & \text { P } & \text { A } & \text { I } & \text { R } & \text { E } & \text { F } & \text { E } & \text { R } & \text { E } & \text { N } & \text { C } & \text { E } & \boldsymbol{S}\end{array}$}

1. Oberg K. Circulating biomarkers in gastroenteropancreatic neuroendocrine tumours. Endocr Relat Cancer 2011;18(1):17-25. DOI: 10.1530/ERC-10-0280.

2. Modlin I.M., Gustafsson B.I., Moss S.F. et al. Chromogranin A biological function and clinical utility in neuroendocrine tumor disease. Ann Surg Oncol 2010;17(9):2427-43. DOI: $10.1245 / \mathrm{s} 10434-010-1006-3$.

3. Aluri V., Dilon J.S. Biochemical testing in neuroendocrine tumors. Endocrinol Metab Clin North Am 2017;46(3):669-77. DOI: $10.1016 /$ j.ecl.2017.04.004.

4. Vinik A.I., Silva M.P., Woltering E.A. et al. Biochemical testing for neuroendocrine tumors. Pancreas 2009;38(8):876-89. DOI: 10.1097/MPA.0b013e3181bc0e77.

5. Pulvirenti A., Rao D., Mcintyre C.A. et al. Limited role of Chromogranin A as clinical biomarker for pancreatic neuroendocrine tumors. HPB (Oxford) 2019;21(5):612-8. DOI: $10.1016 /$ j.hpb.2018.09.016.

6. Cheng Y., Sun Z., Bai C., Yan X. et al. Serum chromogranin A levels for the diagnosis and follow-up of welldifferentiated non-functioning neuroendocrine tumors. Tumour Biol 2016;37(3):2863-9.

DOI: $10.1007 / \mathrm{s} 13277-015-4114-7$.

7. Jun E., Kim S.C., Song K.B. et al. Diagnostic value of chromogranin A in pancreatic neuroendocrine tumors depends on tumor size: A prospective observational study from a single institute. Surgery 2017;162(1):120-30. DOI: 10.1016/j.surg.2017.01.019.

8. Cheng Y., Sun Z., Bai C. et al. Serum chromogranin A levels for the diagnosis and follow-up of well-differentiated non- functioning neuroendocrine tumors. Tumour Biol 2016;37(3):2863-9. DOI: $10.1007 / \mathrm{s} 13277-015-4114-7$.

9. Ma Z.Y., Gong Y.F., Zhuang H.K. et al. Pancreatic neuroendocrine tumors: a review of serum biomarkers, staging, and management. World J Gastroenterol 2020;26(19):2305-22.

DOI: $10.3748 /$ wjg.v26.i19.2305.

10. Любимова Н.В., Чурикова Т.К., Кушлинский Н.Е. Хромогранин А - биохимический маркер нейроэндокринных опухолей. Бюллетень экспериментальной биологии и медицины 2015;160(1):657-60. [Lyubimova N.V., Churikova T.K., Kushlinsky N.E. Chromogranin A is a biochemical marker of neuroendocrine tumors. Byulleten' eksperimental'noj biologii i mediciny $=$ Bulletin of Experimental Biology and Medicine 2015;160(1):657-60. (In Russ.)]. DOI: $10.1007 / \mathrm{s} 10517-016-3254-0$.

11. Любимова Н.В., Томс М.Г., Чурикова Т.К., Харитиди Т.Ю. Клиническое значение хромогранина А при нейроэндокринных опухолях желудочно-кишечного тракта. Альманах клинической медицины 2015;41:35-9. [Lyubimova N.V., Toms M.G., Churikova T.K., Haritidi T.Y. Clinical significance of chromogranin A in neuroendocrine tumors of the gastrointestinal tract. Al'manah klinicheskoj mediciny $=$ Almanac of Clinical Medicine 2015;41:35-9. (In Russ.)]. DOI: 10.18786/2072-0505-2015-41-35-39.

12. Campana D., Nori F., Piscitelli L. et al. Chromogranin A: is it a useful marker of neuroendocrine tumors? J Clin Oncol 2007;25(15):1967-73. DOI: $10.1200 / J C O .2006 .10 .1535$.
13. Stridsberg M., Eriksson B., Fellstrom B. et al. Measurement of chromogranin B can serve as a complement to chromogranin A. Regulatory Peptides 2007;193:80-3. DOI: 10.1016/j.regpep.2006.10.008.

14. Stridsberg M., Oberg K., Li Q. et al. Measurment of chromogranin A, chromogranin B (secretogranin I), chromogranin $\mathrm{C}$ (secretogranin II) and pancreastatin in plasma and urine from patients with carcinoid tumours and endocrine pancreatic tumours. Journal of endocrinology 1995;144(1):49-59. DOI: $10.1677 /$ joe. 0.1440049 .

15. Miki M., Tetuhide I., Hijoka M. et al. Utility of chromogranin B as a biomarker in Japanese patients with pancreatic neuroendocrine tumors. Jpn J Clin Oncol 2017;47(6):520-8. DOI: $10.1093 / \mathrm{jjco} / \mathrm{hyx} 032$.

16. Bech P.R., Martin N.M., Ramachandran R., Bloom S.R. The biochemical utility of chromogranin $\mathrm{A}$, chromogranin $\mathrm{B}$ and cocaine - and amphetamine-regulated transcript for neuroendocrine neoplasia. Ann Clin Biochem 2014;51(1):8-21. DOI: $10.1177 / 0004563213489670$.

17. Любимова Н.В., Тимофеев Ю.С., Лебедева А.В., Кушлинский Н.Е. Хромогранин А и хромогранин В при нейроэндокринных опухолях поджелудочной железы. Медицинский алфавит 2020;(8):26-8. [Lyubimova N.V., Timofeev Yu.S., Lebedeva A.V., Kushlinsky N.E. Chromogranin A and chromogranin $\mathrm{B}$ in neuroendocrine tumors of the pancreas. Medicinskij alfavit $=$ Medical Alphabet 2020;8:26-8. (In Russ.)]. DOI: $10.33667 / 2078-5631-2020-8-26-28$

Вклад авторов

Н.В. Любимова: разработка дизайна исследования, написание текста статьи;

Ю.С. Тимофеев: проведение иммуноферментного анализа, статистический анализ, написание текста статьи;

А.В. Лебедева: сбор и обработка биологического материала, написание текста рукописи;

Н.Е. Кушлинский: разработка дизайна исследования, редактирование текста статьи.

Authors' contributions

N.V. Lyubimova: developed the study design, article writing;

Yu.S. Timofeev: immunoenzyme assay perfoming, statistical analysis, article writing;

A.V. Lebedeva: collecting and processing of biological materials, article writing;

N.E. Kushlinsky: developed the study design, text editing.

ORCID авторов / ORCID of authors

H.В. Любимова / N.V. Lyubimova: https://orcid.org/0000-0003-0430-2754

Ю.С. Тимофеев / Yu.S. Timofeev: https://orcid.org/0000-0001-9305-6713

А.В. Лебедева / A.V. Lebedeva: https://orcid.org/0000-0001-5881-1795

H.Е. Кушлинский / N.E. Kushlinsky: https://orcid.org/0000-0002-3898-4127

Конфликт интересов. Авторы заявляют об отсутствии конфликта интересов. Conflict of interest. The authors declare no conflict of interests.

Финансирование. Исследование проведено без спонсорской поддержки.

Financing. The study was performed without sponsorship.

Соблюдение прав пациентов и правил биоэтики. Все пациенты подписали информированное согласие на участие в исследовании.

Compliance with patient rights and principles of bioethics. All patients gave written informed consent to participate in the study.

Статья поступила: 09.06.2021. Принята к публикации: 07.09.2021.

Article submitted: 09.06.2021. Accepted for publication: 07.09.2021. 